\title{
Estimation System of Exterior Ballistic Measuring Data from Space Tracking Ships
}

\author{
Huifen $\mathrm{Li}^{1, \mathrm{a}}$, You $\mathrm{Wu}^{2, \mathrm{~b}}$, Liwei Zhu ${ }^{1, \mathrm{c}}$ \\ ${ }^{1}$ Joint Lab of Flight Vehicle Ocean-based Measurement and Control, CSMTC, Jiangyin China \\ ${ }^{2}$ College of Science, Hohai University, Naijing China \\ awylhf@yeah.net, ${ }^{b} k j 1906 @$ sina.com, ${ }^{c}$ kjl901@sina.com
}

Keywords: tracking telemetry and command (TT\&C); exterior ballistic measuring; accuracy analysis; performance evaluation; statistical evaluation; shipborne equipment.

\begin{abstract}
To keep abreast of the state changes of the shipborne equipments and the accuracy of the measuring data , an idea that we should build up the quality estimation system of space tracking ships' exterior measuring data is put forward. In which many methods have been taken into consideration, such as cartography, calculus of differences, statistical analysis and comparative analysis of random errors and so on. Evaluate the working state, performance and accuracy of the shipborne exterior ballistic measuring equipment by qualitative assessments and quantitative assessments, meanwhile the accuracy of the calibration parameter is checked as well. The application of the quality estimation system is an effective way to know the problems influencing the accuracy of the calibration parameter directly like finding out the changes of the equipment state and software design flaws.
\end{abstract}

\section{Introduction}

The tracking ship is an athletic platform, so the measuring at sea is carried out under dynamic condition. When the ship is sailing, swaying, whirling and shaking, all these factors will influence the working state and the performance. The shipborne radar drifts with the tracking ship chronically as well, so the zero value and the calibration parameter change[1]. Which influence the precision directly. With the aim of keeping in touch with the performance of the shipborne measuring machine, being sure about the accuracy of the calibration parameter so the measuring data is of high precision, Evaluate the state as well as the performance of the equipments and check the the accuracy of the calibration parameter instantly is of great importance. Therefore, an idea that we should build up the quality estimation system of space tracking ships' exterior measuring data is put forward.

\section{Estimation System of Exterior Ballistic Measuring Data}

\section{Items and content.}

This system evaluates the initial items measured by the shipborne exterior ballistic equipments, as well as the parameters calculated by the center machine. The shipborne exterior ballistic equipments can be divided into two parts, the main part and the auxiliary part. The main part consists of radar and microwave unified TT\&C system, such as USB, UCB and UXB. Whereas the auxiliary part is made up of inertial navigation system and deformation measuring system[2].

(1)The estimation of inertial navigation system.

Evaluate the time system, working state, whether measure and record the data well. Paint the graph of the data measured by the inertial navigation system. Evaluate the quality of the data with observational method.

(2)The estimation of deformation system.

Evaluate the time system, working state, whether measure and record the data well. Paint the graph of the data measured by the deformation system. Evaluate the quality of the data with observational method. 
(3)The estimation of TT\&C equipment(including radar, USB, UCB, UXB),evaluating items.

a) Check the time system, working state and whether we measure and record the data well.

b) Draw the curve. The curve of first-order difference and second-order difference. Evaluate the state of the equipments preliminarily by drawing curves and using difference method.

c) Count up the random error, evaluate the quality of the data statistically.

d) Utilize the method, statistical comparison, to evaluate the accuracy of measurement data[3]. Calculate the residual's random error systematic error and the total error,evaluate the accuracy of the shipborne equipment statistically as well.

(4)The estimation of the central computer's software.

Compared with the instantaneous ballistic trajectory, paint the curve diagram, calculate the random error,systematic error and the total error of the residual, evaluate the accuracy of the central computer's data.

(5)The estimation of spacecraft orbit determination a ccuracy.

a) Calculate the orbital elements before and after the satellite separate from the launch rocket, by using radar USB and UCB, analyse the accuracy orbital elements, evaluate central computer software's accuracy of the orbit determination.

b) Calculate the orbital elements by using the data measured by USB and UCB on the orbiting phase.Analyze the orbit determination of the shipborne equipment,as well as the outer accordance accuracy of the improvement of orbit from the TT\&C.[4]

\section{Flow path of the evaluation.}

To evaluate the exterior ballistic measuring data, the flow path of the evaluation includes prepare for the evaluation, data preprocessing, data evaluation and have an evaluation report. Specific procedures are as follows.

Step 1 Prepare the evaluation. Preparing the evaluation includes modify the software we use toevaluate, collect the observation, prepare the parameters and so on.

Step 2 Data preprocessing. Data preprocessing means choose effective observation, data conversion, justifiability inspection of data, parameter zero time correction, delay correction, correction of axis error, to get the data of the inertial horizontal coordinate system.

Step 3 Data Evaluation.With the methods like cartography, calculus of differences, error analysis method, statistical comparison to evaluate the observation measured by TT\&C equipments.

Step 4 Have Evaluation Reports.Have evaluation reports via various TT\&C equipments.

\section{Technical support}

\section{Evaluation methods.}

Evaluate the work from performance evaluation and statistical evaluation, using the methods like cartography, calculus of differences, error analysis method, statistical comparison and so on. Performance evaluation includes two methods, cartography and calculus of differences. Statistical evaluation includes two method as well, error analysis and statistical comparison[3].

(1) Cartography

Paint the curve diagram of the observation and then get the changes in two adjacent datum, then we know whether the outlier exist. Then judge whether the change is normal, it is abnormal if there exist phenomena like lose the target, "convex", "re-entrant”, and "step".

(2)Calculus of Differences

Make first-order difference $\Delta x_{i}=x_{i+1}-x_{i}(i=1,2, \cdots, n-1)$ on the observation $x_{1}, x_{2}, \cdots, x_{n}$ first, then calculate standard $S_{x}$ deviation and mean value of difference $\Delta \bar{X}$ of the observation. If the value of difference $\Delta X^{\prime}$ satisfy.

$$
\frac{\left|\Delta X^{\prime}-\Delta \bar{X}\right|}{\sqrt{2} S_{x}}>k
$$


then it is outlier, otherwise not. Difference method is a good way to evaluate when the truth value and the error has not be separated, the observation is first-order difference which is equal to velocity, whose variable quantity is quite small, so it is easy to find the outlier. But if the truth value of the derivative is large, we can judge it by using second-order difference or third-order difference, the interrelated formula is.

$$
\frac{\left|\Delta^{2} X^{\prime}-\Delta^{2} \bar{X}\right|}{\sqrt{6} S_{x}}>k, \frac{\left|\Delta 3 X^{\prime}-\Delta^{3} \bar{X}\right|}{\sqrt{20} S_{x}}>k
$$

based on the principle of error propagation, $\sigma_{\Delta x}=\sqrt{2} \sigma_{x}, \sigma_{\Delta^{2} x}=\sqrt{6} \sigma_{x}, \quad \sigma_{\Delta^{3} x}=\sqrt{20} \sigma_{x}$. We always value $\mathrm{k} 3$ or $4 . \sigma_{\mathrm{x}}$ is the root mean squared deviation of observation.

(3)The Statistical Method of the Random Error

Random error is a great index to know the equipment characteristic and the quality of the data, we count random error by using variate difference method and least square residual fitting method.

a) Variate difference method

People usually consider that exterior ballistic measuring data is an unbroken curve which is changed with time, the curve can be showed as certain order's polynomial as well. We can remove trends(true information and systematic error) in the observation through difference processing, then the random variable in the observation will be resolved as well. Variate difference method is one of the most popular way we have used to collect random error. Variate difference method has simple principle and easy calculation, furthermore,no extra information is needed when collecting[5]. The formula we used to collect the random error by variate difference method is as follows:

$$
\sigma_{Y}=\sqrt{\frac{(p !)^{2}}{(2 p) !(n-p L)} \sum_{j=1}^{n-p L}\left(\sum_{k=0}^{p}(-1)^{k}\left(\frac{p !}{k !(p-k) !}\right) Y_{p L+j-k L}\right)^{2}}
$$

$\sigma_{Y}$ is random error, $p$ is difference error, $\mathrm{n}$ is total data points, $\mathrm{L}$ is difference step, $\left\{Y_{j}\right\}_{j=1,2, \cdots, n}$ are the collected observation.

Variate difference method is only suitable when the data's random error is flat noise.

b) Least square residual fitting method.

A time polynomial can be used to describe observation. We can get the random error with the least square method, fitting to get the polynomial of the observation and collect the fitting residual. As what has been told above, one of the basic assumptions of variate difference method is collecting the deviation independently, but least square residual fitting method does not have any extra requirements on whether the data is independent, so it has a better applicability and better accuracy. But we should take ship swaying into consideration, the data used in the telemetering coordinate system is processed with variate difference method. On the other way, we use least square residual fitting method when we are correcting the ship swaying deviation, changing the coordinates, and in the inertial horizontal coordinate system.

(4) Statistical comparison method

Statistical comparison method is a common way to diagnose and analyze the quality of observation and evaluate the accuracy of data processing model. It includes three methods, 'observation comparison', 'missile trajectory comparison' and 'using orbital elements to get observation comparison'.

a) Observation comparison

Arrange two sets of equipments to track in the same observation arc simultaneously, then we are able to compare one observation from the other and get the state information of the appraisal equipment which is included in the residual error. Comparing different observation is a way to resolve equipment's residual error and judge the quality of the observation. Residual error can be used to evaluate the accuracy of the observation and analyse outlier.

b) Missile trajectory comparison

When evaluating the observation of tracking launch rocket, we can compare the missile trajectory, consider the reference information like ideal theoretically ballistic trajectory, the ballistic trajectory of the launch rocket measured by exterior ballistic measuring equipment or the telemetry equipment. 
By comparison, evaluate the consistency of ideal ballistic trajectory and theoretically ballistic trajectory or any reference ones got by other ways statistically.

c) Using orbital elements to get observation comparison

If the orbital element is a fusion calculated by lots of observation from sets of TT\&C equipments, or just measured by high-precision optical tracking system, then it should be transformed into the same type of data as observation before comparison, the residual we get can be used to evaluate the accuracy of the equipment.

Utilize the residual to figure out the systematic error, random error and the total error, with all the things above, we will finish the data quality evaluation.

\section{Evaluation principle.}

As to the quality of the data measured by the ancillary equipment, we can evaluate the performance according to the changing rules and the changing cycle. When it comes to the data quality of the main equipment, we can combine three different methods, graphical analysis, error analysis and statistical comparison together to do the statistical evaluation.

We can tell whether the changes of the observation is normal according to the changing rules of the chart, utilizing statistic methods to know the error and compare it with the equipment's index, by which we will know whether the general measurement precision is out of the standard. Meanwhile, according to the time system, the state of the equipment, data recording and the permillage of outlier inobservation, we can tell the quality of the data furthermore. If the general measurement precision of the observation is within the index of the equipment, and the outlier of the observation is smaller than $3 \%$, then the observation is normal. If the general measurement precision of the observation is close to the index, and the outlier is within $45 \%$,the observation is basically normal. If the general measurement precision is beyond index, meanwhile, the outlier is beyond $45 \%$, the observation is abnormal. If the general measurement precision of the observation is fall more larger than the index, the outlier of the observation is larger than $100 \%$ as well, then there is serious problem with the observation.

\section{Conclusions}

In the rendezvous and docking mission, the exterior ballistic measuring data evaluation system of the tracking ship did really well. Such as finding an equipment problem 'USB's optical axis began to drift' through the phenomenon 'the system error of azimuth went beyong index'. For the time being, the evaluation system has become an effective way to check the shipborne equipment's state, performance and evaluate the quality of the data. Along with the exterior ballistic measuring data evaluation system take effect in the spacecrafts missions, it has proved itself a practical system.

\section{References}

[1] ZHONG Dean. Technology of Calibration and Flight Test for TT\&C Ship’s Measuring Communicating Equipment[M].Beijing:National Defense Industry Press,2009:138-172.

[2] JIANG Wenda.Space TT\&C Ship[M].Beijing:National Defense Industry Press,2002:78-146.

[3] LI Huifen,ZHANG Zhonghua.The Exterior Tracking \& Measuring Data Analyzing ing and Processing of Instrumentation Ship[J].Journal of Spacecraft TT\&C Technology, 2008, 27(6):65-70.

[4] Kong Deyong. Influence of Ship’s Position Error on Exterior Trajectory Mesaurement and Orbit Determination[J].Telecommunication Engineering,2010,50(9):106-109.

[5] HU Shaolin,XU Aihua,GUO Xiaohong.Data Processing Technology Applied to Pulse Radar Traching[M].Beijing:National Defense Industry Press,2007:85-121. 